\title{
Correction to: On maximum of Gaussian random fields having unique maximum point of its variance
}

\section{Sergey G. Kobelkov ${ }^{1}$. Vladimir I. Piterbarg ${ }^{1,2} \cdot$ Igor V. Rodionov $^{3}$}

Published online: 20 November 2020

(C) Springer Science+Business Media, LLC, part of Springer Nature 2020

Correction to: Extremes (2019) 22: 413-432

https://doi.org/10.1007/s10687-019-00346-2

In the proof of Proposition 3 below formula (31), it was stated that $\Sigma(u)$ and $\Sigma^{\prime}(u)$ are integral sums for the integral

$$
I(u)=\int_{f(\mathbf{t}) \leq u^{-1} \gamma_{1}(u)} e^{-u^{2} f(\mathbf{t})} d \mathbf{t},
$$

where $f(\mathbf{t})=\left(1-\sigma^{2}(\mathbf{t})\right) / 2$. In spite of the fact that $\Sigma^{\prime}(u) \leq I(u) \leq \Sigma(u)$, the relation $\Sigma(u) / \Sigma^{\prime}(u) \rightarrow 1, u \rightarrow \infty$, was not justified. To show this, we should significantly change the proof of Proposition 3.

First, we modify Condition 5.

The online version of the original article can be found at https://doi.org/10.1007/s10687-019-00346-2.

Igor V. Rodionov

vecsell@gmail.com

Sergey G. Kobelkov

sergeyko81@gmail.com

Vladimir I. Piterbarg

piter@mech.math.msu.su

1 Lomonosov Moscow State University, Moscow, Russia

2 International Laboratory of Stochastic Analysis and its Applications National Research

University Higher School of Economics, Moscow, Russia

3 Trapeznikov Institute of Control Sciences, Russian Academy of Sciences, Profsoyuznaya 65, 117997 Moscow, Russia 
Condition $5^{*}$ For any $\mathbf{t}$ there exists the function $h_{1}(\mathbf{t}) \in[0, \infty]$ such that

$$
\lim _{u \rightarrow \infty} u^{2}\left(1-\sigma^{2}(\mathbf{q}(u) \mathbf{t})\right)=h_{1}(\mathbf{t}) .
$$

Moreover, if $h_{1}(\mathbf{e})=0$ for all $\mathbf{e} \in \mathbb{S}^{d-1}$, the unit sphere in $\mathbb{R}^{d}$, then

$$
\lim _{u \rightarrow \infty} u^{2}\left(\sigma^{2}(\mathbf{s})-\sigma^{2}(\mathbf{s}+\mathbf{q}(u) \mathbf{t})\right)=0
$$

uniformly in $\mathbf{t}$ from any closed set and $\mathbf{s} \in \mathbb{B}_{\varepsilon}$.

Let the latter assumption hold. Then, using the argument similar to the one on the regular variation property of $1-r(\mathbf{t})$ (see page 416 in the original paper), we can prove that $h_{1}(\mathbf{t})$ is continuous and that if $h_{1}(\mathbf{e}) \in(0, \infty)$ for some vector $\mathbf{e} \in \mathbb{R}^{d}$ then $h_{1}(t \mathbf{e})$ is regular varying at zero. Thus the condition " $h_{1}(\mathbf{e})=0$ for all $\mathbf{e} \in \mathbb{S}^{d-1}$ " implies that $h_{1}(\mathbf{t})=0$ for all $\mathbf{t} \in \mathbb{R}^{d}$ and then the last condition may be replaced by the condition " $h_{1}(\mathbf{e})=0$ for all $\mathbf{e} \in \mathbb{S}^{d-1}$ " in the statement of Proposition 3 .

Additionally, note that we need the second part of Condition $5^{*}$ to justify that the sets $\left\{B_{k}(u)\right\}_{k \geq 0}$ appearing below satisfy the assumptions of Theorem 1 and to apply the standard argument for evaluating the double sum (see the relation (9) below). It might seem that we can replace the second part of Condition $5^{*}$ with the following weaker assumption in spirit of the condition E4, Piterbarg and Rodionov (2020),

Moreover, if $h_{1}(\mathbf{e})=0$ for all $\mathbf{e} \in \mathbb{S}^{d-1}$, then Eq. 2 holds uniformly in $\mathbf{t}$ from any closed set and there exist $c>0$ and $K<\infty$ such that for every $x \in[0, c], \mathbf{t}_{0} \in \mathbb{R}^{d}$ and $\mathbf{e} \in \mathbb{S}^{d-1}$, the number of roots of the equation $1-\sigma^{2}\left(\mathbf{t}_{0}+y \mathbf{e}\right)=x$ with respect to $y$ does not exceed $K$.

The analogue of this assumption was used to examine the stationary-like case in the proof of Theorem 1, Piterbarg and Rodionov (2020). The exact value of $K$ does not play a role, but its finiteness guarantees the existence of "large" subintervals $B_{k}^{\prime}(u)$ satisfying the assumptions of Theorem 1 of the original paper (see this argument on p. 4868, Piterbarg and Rodionov, 2020), and uniformity allows one to show asymptotic negligence of the double sum. Although this assumption is enough to prove Proposition 3 in one-dimensional case, the distance between two nonneighboring sets $B_{k}(u)$ and $B_{l}(u)$ can be arbitrarily small in multi-dimensional case even if $K=2$. Due to this fact it is not possible to show that the double sum is asymptotically smaller than the single sum under the latter assumption (see the relations (8), (9) below).

Recall that in the proof of Proposition 3 we deal with the simplified model $X(\mathbf{t})=$ $X_{0}(\mathbf{t}) \sigma(\mathbf{t}), \mathbf{t} \in S$, satisfying Conditions $1-4$ and $5^{*}$, where $X_{0}(\mathbf{t})$ is a homogeneous Gaussian field with mean zero and covariance function satisfying Condition 4 and $\sigma(\mathbf{t})$ satisfies Condition $5^{*}$.

Take small $\varepsilon_{+}$and $\varepsilon_{-}$with $\varepsilon_{+}>\varepsilon_{-}>0$. For sufficiently large $u_{0}$ and all $u \geq u_{0}$ there exists $\varepsilon_{0}=\varepsilon_{0}(u) \in\left(\varepsilon_{-}, \varepsilon_{+}\right)$with $u \gamma_{1}(u) / \varepsilon_{0} \in \mathbb{N}$. We use this $\varepsilon_{0}$ below. Denote

$$
B_{k}(u)=\left\{\mathbf{t}: k \varepsilon_{0} \leq u^{2}\left(1-\sigma^{2}(\mathbf{t})\right)<(k+1) \varepsilon_{0}\right\},
$$

for all $k \geq 0$ with $B_{k}(u) \subset B_{u}$.

Recall that for all $\mathbf{t} \neq \mathbf{0}$

$$
\lim _{u \rightarrow \infty} \frac{u^{2}\left(1-\sigma^{2}(\mathbf{q}(u) \mathbf{t})\right)}{u^{2}(1-r(\mathbf{q}(u) \mathbf{t}))}=0 .
$$


Moreover, by the second part of Condition $5^{*}$ we have for all $\mathbf{t} \in \mathbb{R}^{d}$ and $\mathbf{s} \in \mathbb{B}_{\varepsilon}$

$$
\lim _{u \rightarrow \infty} \frac{u^{2}\left(\sigma^{2}(\mathbf{s})-\sigma^{2}(\mathbf{s}+\mathbf{q}(u) \mathbf{t})\right)}{u^{2}(1-r(\mathbf{q}(u) \mathbf{t}))}=0 .
$$

Now let us prove that there exist functions $Q_{i}(u), i=1, \ldots, d$, such that

$$
Q_{i}(u) / q_{i}(u) \rightarrow \infty, \quad u \rightarrow \infty,
$$

and

$$
u^{2}\left(\sigma^{2}(\mathbf{s})-\sigma^{2}(\mathbf{s}+\mathbf{Q}(u) \mathbf{t})\right) \rightarrow 0, \quad u \rightarrow \infty,
$$

uniformly in $\mathbf{t} \in E^{d}=[-1,1]^{d}$ and $\mathbf{s} \in \mathbb{B}_{\varepsilon}$. Denote

$$
R(u):=\sup _{\mathbf{t} \in E^{d}, \mathbf{s} \in \mathbb{B}_{\varepsilon}} \frac{\sigma^{2}(\mathbf{s})-\sigma^{2}(\mathbf{s}+\mathbf{q}(u) \mathbf{t})}{1-r(\mathbf{q}(u) \mathbf{t})} .
$$

Since $q_{i}(u)$ are monotone for every $i, i=1, \ldots, d$ (see the corresponding argument between the relations (5) and (6) of the original paper), $R(u)$ is monotone as well and tends to 0 due to uniform convergence in $\mathbf{t} \in E^{d}$ in (4).

We will look for $Q_{i}(u)$ in the form $Q_{i}(u)=q_{i}(f(u)), i=1, \ldots, d$, where $f(u) \rightarrow \infty$ and $f(u) / u \rightarrow 0$ as $u \rightarrow \infty$. In this case (5) follows by regular variation property of $q_{i}(u)$ for all $i$. Assume that

$$
\frac{u^{2}}{f^{2}(u)} R(f(u)) \rightarrow 0, \quad u \rightarrow \infty .
$$

From the latter and Condition 4 we have for some $\Delta>0$

$$
\begin{aligned}
\sup _{\mathbf{t} \in E^{d}, \mathbf{s} \in \mathbb{B}_{\varepsilon}} \frac{\sigma^{2}(\mathbf{s})-\sigma^{2}(\mathbf{s}+\mathbf{Q}(u) \mathbf{t})}{1-r(\mathbf{q}(u) \mathbf{t})} & \leq \sup _{\mathbf{t} \in E^{d}, \mathbf{s} \in \mathbb{B}_{\varepsilon}} \frac{\sigma^{2}(\mathbf{s})-\sigma^{2}(\mathbf{s}+\mathbf{Q}(u) \mathbf{t})}{(1-\Delta) \frac{f^{2}(u)}{u^{2}}(1-r(\mathbf{Q}(u) \mathbf{t}))} \\
& =(1-\Delta)^{-1} \frac{u^{2}}{f^{2}(u)} R(f(u)) \rightarrow 0
\end{aligned}
$$

as $u \rightarrow \infty$. Thus, the latter and Condition 4 imply (6).

It immediately follows from Eq. 6 and the definition of $B_{k}(u)$, that for every $k \geq 0$ and sufficiently large $u$ there exists $\mathbf{t}_{k}=\mathbf{t}_{k}(u) \in B_{k}(u)$ (without loss of generality we can select $\mathbf{t}_{k}$ with $\left.u^{2}\left(1-\sigma^{2}\left(\mathbf{t}_{k}\right)\right)=(k+1 / 2) \varepsilon_{0}\right)$ such that

$$
\mathbf{t}_{k}+\bigotimes_{i=1}^{d}\left[-Q_{i}(u), Q_{i}(u)\right] \subset B_{k}(u) .
$$

Thus for every $k \geq 0$ the set $B_{k}(u)$ satisfies the assumptions of Theorem 1 . The last condition in this theorem holds for all $\delta>0$ and $k$ since $\left|B_{u}\right| \rightarrow 0$ as $u \rightarrow \infty$, where $|\cdot|$ denotes the volume. Hence, for every $k \geq 0$

$$
\mathbf{P}\left(\max _{\mathbf{t} \in B_{k}(u)} X_{0}(\mathbf{t})>u\right)=(1+\delta(u)) H_{\mathbf{q}}\left|B_{k}(u)\right| \prod_{i=1}^{d} q_{i}^{-1}(u) \Psi(u),
$$

where $\delta(u) \rightarrow 0$ as $u \rightarrow \infty$.

The remaining part of the proof almost coincides with the corresponding part of the proof of Theorem 1, Piterbarg and Rodionov (2020), see Section 3.5 there. We 
just indicate the differences. The main difference is that due to Condition $5^{*}$ all sets $B_{k}(u), k \geq 0$, are "large", namely, satisfy the assumptions of Theorem 1 , and this observation makes the proof much simpler.

Note, that the sets of indices $\mathcal{L}=\left\{k: B_{k}(u) \cap B_{u} \neq \varnothing\right\}$ and $\mathcal{L}^{\prime}=\left\{k: B_{k}(u) \subset\right.$ $\left.B_{u}\right\}$ coincide due to the choice of $\varepsilon_{0}$. Thus by Bonferroni inequality

$$
P\left(B_{u}, u\right) \leq \sum_{k \in \mathcal{L}} \mathbf{P}\left(\max _{\mathbf{t} \in B_{k}(u)} X(\mathbf{t})>u\right)
$$

and

$$
\begin{aligned}
P\left(B_{u}, u\right) \geq & \sum_{k \in \mathcal{L}} \mathbf{P}\left(\max _{\mathbf{t} \in B_{k}(u)} X(\mathbf{t})>u\right) \\
& -\sum_{k \in \mathcal{L}} \sum_{l \neq k, l \in \mathcal{L}} \mathbf{P}\left(\max _{\mathbf{t} \in B_{k}(u)} X(\mathbf{t})>u, \max _{\mathbf{s} \in B_{l}(u)} X(\mathbf{s})>u\right) .
\end{aligned}
$$

Denote

$$
\sigma_{k}=\sqrt{1-u^{-2} k \varepsilon_{0}}, \quad u_{k}=u / \sigma_{k}, \quad k \geq 0 .
$$

Probabilities under the single sum in Eqs. 8 and 9 are bounded from above and from below by probabilities of events

$$
A_{k}(u)=\left\{\max _{\mathbf{t} \in B_{k}(u)} X_{0}(\mathbf{t})>u_{k}\right\} \quad \text { and } \quad A_{k}^{\prime}(u)=\left\{\max _{\mathbf{t} \in B_{k}(u)} X_{0}(\mathbf{t})>u_{k+1}\right\},
$$

respectively. We have by Eq. 7 for $k \in \mathcal{L}$

$$
\mathbf{P}\left(A_{k}\right)=\left(1+\delta\left(u_{k}\right)\right) H_{\mathbf{q}}\left|B_{k}(u)\right| \prod_{i=1}^{d} q_{i}^{-1}\left(u_{k}\right) \Psi\left(u_{k}\right),
$$

and

$$
\mathbf{P}\left(A_{k}^{\prime}\right)=\left(1+\delta\left(u_{k+1}\right)\right) H_{\mathbf{q}}\left|B_{k}(u)\right| \prod_{i=1}^{d} q_{i}^{-1}\left(u_{k+1}\right) \Psi\left(u_{k+1}\right) .
$$

Denote

$$
\Sigma(u)=\sum_{k \in \mathcal{L}}\left|B_{k}(u)\right| e^{-k \varepsilon_{0} / 2}, \quad \Sigma^{\prime}(u)=\sum_{k \in \mathcal{L}}\left|B_{k}(u)\right| e^{-(k+1) \varepsilon_{0} / 2} .
$$

Using the same argument as in the corresponding part of the proof of Theorem 1, given in Section 3.5, Piterbarg and Rodionov (2020), we have

$$
\begin{aligned}
& \sum_{k \in \mathcal{L}} \mathbf{P}\left(A_{k}\right) \leq\left(1+\delta_{1}(u)\right) \frac{H_{\mathbf{q}}}{\sqrt{2 \pi} u \prod_{i=1}^{d} q_{i}(u)} e^{-u^{2} / 2} \Sigma(u), \\
& \sum_{k \in \mathcal{L}} \mathbf{P}\left(A_{k}^{\prime}\right) \geq\left(1-\delta_{1}(u)\right) \frac{H_{\mathbf{q}}}{\sqrt{2 \pi} u \prod_{i=1}^{d} q_{i}(u)} e^{-u^{2} / 2} \Sigma^{\prime}(u),
\end{aligned}
$$

where $\delta_{1}(u) \rightarrow 0$ as $u \rightarrow \infty$. Clear, by Eq. $1, \Sigma^{\prime}(u) \leq I(u) \leq \Sigma(u)$ and

$$
\Sigma(u) / \Sigma^{\prime}(u)=e^{\varepsilon_{0} / 2} \leq 1+2 \varepsilon_{+}
$$


for all sufficiently small $\varepsilon_{+}$. Thus, we deduce that $\Sigma(u)$ and $\Sigma^{\prime}(u)$ are the upper and lower integral sums for the integral $I(u)$, respectively. It remains to be noted that $L_{f}\left(u^{2}\right)=I(u)(1+o(1))$ as $u \rightarrow \infty$.

Since we use the sets $\left\{B_{k}(u)\right\}_{k \geq 0}$ in our note instead of the boxes $\left\{\Delta_{\mathbf{k}}\right\}_{\mathbf{k} \in \mathbb{Z}^{d}}$ in the original paper, we should revise the second part of the proof of Proposition 3 as well. Let us estimate from above the double sum in Eq. 9. First we consider nonneighboring pairs of sets. Rewriting the relation (32) in the original paper for $\left\{B_{k}(u)\right\}_{k \geq 0}$ and following the steps below this relation, we have for $k, l \geq 0$ with $|k-l|>1$

$$
\begin{aligned}
\mathbf{P}\left(A_{k} \cap A_{l}\right) & \leq \mathbf{P}\left(\max _{(\mathbf{s}, \mathbf{t}) \in B_{k}(u) \otimes B_{l}(u)} \frac{X_{0}(\mathbf{s})+X_{0}(\mathbf{t})}{\sqrt{2+2 r(\mathbf{t}-\mathbf{s})}} \geq \frac{u_{k}+u_{l}}{\sqrt{2+2 r_{k, l}(u)}},\right) \\
& \leq C \frac{\left|B_{k}(u)\right| \cdot\left|B_{l}(u)\right|}{\left(\prod_{i=1}^{d} q_{i}(u)\right)^{2}} \Psi\left(\frac{u_{k}+u_{l}}{\sqrt{2+2 r_{k, l}(u)}}\right)
\end{aligned}
$$

where

$$
r_{k, l}(u)=\max _{(\mathbf{s}, \mathbf{t}) \in B_{k}(u) \otimes B_{l}(u)} r(\mathbf{t}-\mathbf{s})
$$

and the constant $C$ does not depend on $k, l$. Note, that

$$
\min _{(\mathbf{s}, \mathbf{t}) \in B_{k}(u) \otimes B_{l}(u)}\left|\sigma^{2}(\mathbf{t})-\sigma^{2}(\mathbf{s})\right|=u^{-2}(|k-l|-1) \varepsilon_{0}
$$

by Eq. 3 and continuity of $\sigma^{2}(\mathbf{t})$, therefore Eq. 4 and Condition 4 implies that $u^{2}(1-$ $\left.r_{k, l}(u)\right) \rightarrow \infty$ as $u \rightarrow \infty$ uniformly in $k, l \geq 0$ with $|k-l|>1$. It follows that there exists an increasing $\kappa(u)$ tending to infinity as $u \rightarrow \infty$ such that for all $k, l \geq 0$ with $|k-l|>1$

$$
u^{2}\left(1-r_{k, l}(u)\right) / \kappa(u) \rightarrow \infty, \quad u \rightarrow \infty .
$$

Similarly to three first lines on page 425 of the original paper, we have

$$
\begin{aligned}
\frac{\left(u_{k}+u_{l}\right)^{2}}{2+2 r_{k, l}(u)} & \geq u^{2}+\frac{1}{2} \kappa(u)+\frac{1}{4} u^{2}\left(1-\sigma_{k}^{2}\right)+\frac{1}{4} u^{2}\left(1-\sigma_{l}^{2}\right) \\
& =u^{2}+\kappa(u) / 2+k \varepsilon_{0} / 4+l \varepsilon_{0} / 4
\end{aligned}
$$

From Eqs. 12 and 13 we derive the following bound for the double sum in Eq. 9

$$
\begin{gathered}
\sum_{k \in \mathcal{L}} \sum_{l \neq k, l \in \mathcal{L}} \mathbf{P}\left(\max _{\mathbf{t} \in B_{k}(u)} X(\mathbf{t})>u, \max _{\mathbf{s} \in B_{l}(u)} X(\mathbf{s})>u\right) \leq \sum_{k \in \mathcal{L}} \sum_{|l-k|>1, l \in \mathcal{L}} \mathbf{P}\left(A_{k} \cap A_{l}\right) \\
\leq C_{1} \frac{1}{\left(\prod_{i=1}^{d} q_{i}(u)\right)^{2}} \exp (-\kappa(u)) \Psi(u)\left(\sum_{k \in \mathcal{L}}\left|B_{k}(u)\right| e^{-k \varepsilon_{0} / 4}\right)^{2}
\end{gathered}
$$

for some $C_{1}>0$, where the last term on the right-hand side can be estimated from above by $I(u)\left(1+\varepsilon_{1}\right)$ for any $\varepsilon_{1}>0$ and sufficiently large $u$ via Schwarz inequality. Therefore, the term $\exp (-\kappa(u))$ in Eq. 14 gives that the double sum over nonneighboring intervals is infinitely smaller than both the single sums in Eqs. 10, 11. 
It remains to consider the asymptotic of the double sum over neighboring sets. Denote

$$
\Upsilon(u, \mathbf{t}):=\mathbf{t}+\bigotimes_{i=1}^{d}\left[-\sqrt{q_{i}(u) Q_{i}(u)}, \sqrt{q_{i}(u) Q_{i}(u)}\right]
$$

and

$$
\partial B_{k}(u)=\left\{\mathbf{t}: u^{2}\left(1-\sigma^{2}(\mathbf{t})\right)=(k+1) \varepsilon_{0}\right\}, \quad k \geq 0 .
$$

Let $l-k=1$ and set

$$
B_{l}^{\prime}(u)=B_{l}(u) \cap\left\{\mathbf{s}: \exists \mathbf{t} \in \partial B_{k}(u) \text { such that } \mathbf{s} \in \Upsilon(u, \mathbf{t})\right\},
$$

the "small neighborhood" of $\partial B_{k}(u)$ in $B_{l}(u)$. We have

$$
\begin{aligned}
\mathbf{P}\left(A_{k} \cap A_{l}\right) \leq & \mathbf{P}\left(\max _{\mathbf{t} \in B_{l}^{\prime}(u)} X_{0}(\mathbf{t})>u_{l}\right) \\
& +\mathbf{P}\left(\max _{(\mathbf{s}, \mathbf{t}) \in B_{k}(u) \otimes\left(B_{l}(u) \backslash B_{l}^{\prime}(u)\right)} X_{0}(\mathbf{s})+X_{0}(\mathbf{t})>u_{k}+u_{l}\right) .
\end{aligned}
$$

The set $B_{l}^{\prime}(u)$ satisfies the assumptions of Theorem 1 for every $l \geq 1$, thus we can use the same argument for estimating the sum of the first probabilities on the righthand side as we used for estimating the single sum in Eqs. 8 and 9 above. This sum is infinitely smaller than the single sum above since we have $\left|B_{l}^{\prime}(u)\right| /\left|B_{l}(u)\right| \rightarrow 0$ as $u \rightarrow \infty$ by Conditions 4 and $5^{*}$. Next, from Eq. 4 it follows that $u^{2}\left(1-r_{k l}^{\prime}(u)\right) \rightarrow \infty$ as $u \rightarrow \infty$ where

$$
r_{k l}^{\prime}(u)=\max _{(\mathbf{s}, \mathbf{t}) \in B_{k}(u) \otimes\left(B_{l}(u) \backslash B_{l}^{\prime}(u)\right)} r(\mathbf{t}-\mathbf{s}) .
$$

Thus the argument of the double sum estimation over nonneighboring sets can be applied for the sum of the second probabilities on the right-hand side of Eq. 15 . Hence this sum is infinitely smaller than the single sum as well.

To end the proof we just repeat the argument given at the top of page 426 of the original paper. The result follows.

Acknowledgments The authors wish to thank Prof. Enkelejd Hashorva, University of Lausanne, for having drawn our attention to the error. The work of Igor Rodionov under the proof of Proposition 3 is partly supported by the Russian Foundation for Basic Research Grant No 19-01-00090 and by Young Russian Mathematics award.

\section{References}

Piterbarg, V.I., Rodionov, I.V.: High excursions of Bessel and related random processes. Stoch. Proc. Appl. 130, 4859-4872 (2020). https://doi.org/10.1016/j.spa.2020.02.002

Publisher's note Springer Nature remains neutral with regard to jurisdictional claims in published maps and institutional affiliations. 